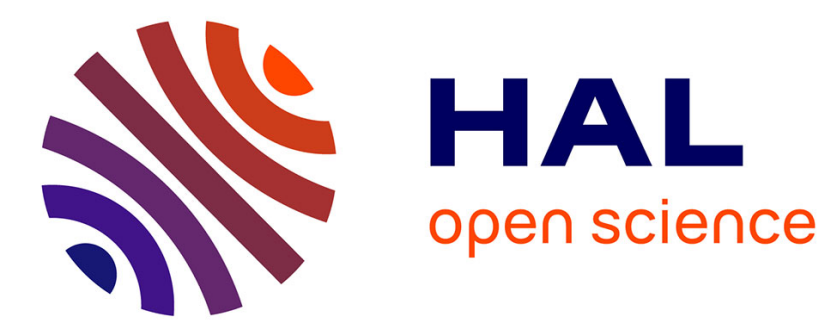

\title{
Quel bilan et quelles perspectives pour l'action des partenariats public-privé dans la régénération de Glasgow ?
}

Fabien Jeannier

\section{- To cite this version:}

Fabien Jeannier. Quel bilan et quelles perspectives pour l'action des partenariats public-privé dans la régénération de Glasgow ?. Observatoire de la société britannique, 2010, 8, pp.147-169. 10.4000/osb.916 . halshs-00956453

\section{HAL Id: halshs-00956453 \\ https://shs.hal.science/halshs-00956453}

Submitted on 6 Mar 2014

HAL is a multi-disciplinary open access archive for the deposit and dissemination of scientific research documents, whether they are published or not. The documents may come from teaching and research institutions in France or abroad, or from public or private research centers.
L'archive ouverte pluridisciplinaire HAL, est destinée au dépôt et à la diffusion de documents scientifiques de niveau recherche, publiés ou non, émanant des établissements d'enseignement et de recherche français ou étrangers, des laboratoires publics ou privés. 


\title{
Quel bilan et quelles perspectives pour l'action des partenariats public-privé dans la régénération de Glasgow?
}

\author{
Fabien Jeannier
}

\section{Résumé}

Depuis le début des années 1980, Glasgow a mis en œuvre une politique de régénération urbaine de grande ampleur dont l'objectif clairement énoncé à cette époque était d'en faire une capitale culturelle et économique de dimension internationale et d'effacer l'image extrêmement négative qui lui était associée, conséquence d'une longue et douloureuse période de déclin. Pour y parvenir, les responsables travaillistes de la ville ont progressivement eu recours aux partenariats public-privé afin de mobiliser des ressources et prendre en charge la régénération des zones les plus défavorisées de la ville. Les sociétés locales de développement furent des exemples de partenariats public-privé qui tentèrent d'aborder sous un angle global les divers aspects de la régénération de la ville, à travers la mise en place de solutions intégrées dont l'objectif était d'intervenir à la fois sur l'ensemble des problématiques économiques, sociales, culturelles des quartiers pauvres de la ville et de leurs populations. L'objectif de cet article est de discuter leurs résultats et les perspectives et limites que laisse entrevoir la modernisation de l'action publique dans le domaine de la régénération urbaine à Glasgow et dans le centre ouest de l'Écosse.

\section{Mots-clés : régénération urbaine, Glasgow, partenariats public-privé, modernisation}

Depuis le début des années 1980, Glasgow a mis en œuvre une politique de régénération urbaine de grande ampleur dont l'objectif clairement énoncé à cette époque était d'en faire une capitale culturelle et économique de dimension internationale. La ville voulait à tout prix effacer l'image extrêmement négative qui lui était associée, conséquence d'une longue et douloureuse période de déclin. Pour permettre ce redéveloppement de la ville, les responsables travaillistes ${ }^{1}$ ont progressivement eu recours aux partenariats entre organismes publics et acteurs privés afin de mobiliser des ressources et prendre en charge une bonne partie de la régénération physique, économique et sociale de la ville. C'est ainsi que sont nées les sociétés locales de développement (local development agencies), résultats de la réflexion menée par la municipalité après le constat d'échec des différentes politiques de régénération menées au fil des décennies, notamment après la seconde guerre mondiale.

Bien que les sociétés locales de développement aient été des exemples de partenariats public-privé particulièrement en vue ${ }^{2}$ qui abordaient sous un angle global les divers aspects de la

\footnotetext{
1 Le contexte de la ville de Glasgow est le suivant : il s'agit d'une ville qui, à deux exceptions près depuis 1933, a toujours été dirigée par les travaillistes. S'il faut donc attendre 1933 pour que les travaillistes prennent le contrôle de la gestion de la ville leur hégémonie à Glasgow n'a connu que deux courtes d'interruptions depuis : entre 1949 et 1951 puis entre 1969 et 1971.

2 Glasgow a souvent été citée comme un cas exemplaire par des institutions telles que l'Union Européenne ou l'OCDE. Une délégation de l'OCDE en visite à Glasgow en janvier 2006 a ainsi félicité la ville pour parvenir à attirer des entreprises majeures de la finance, pour avoir développé une industrie touristique florissante basée sur l'hébergement de conventions et l'organisation de manifestations culturelles et pour être en mesure de proposer une offre commerciale importante. L'OCDE classe Glasgow dans le premier quart des villes similaires au regard des efforts employés à transformer une ville post-industrielle en une ville « adaptée à l'économie moderne » (GCC, 2006, 18).
} 
régénération de la ville dans les quartiers périphériques les plus en difficulté, cette modalité de l'intervention publique dans la régénération de Glasgow a évolué. C'est donc à travers la récente refondation du réseau des sociétés locales de développement en agences locales de régénération (Local Regeneration Agencies) à Glasgow et, parallèlement, la mise en place au cours des quatre ou cinq dernières années par le gouvernement écossais, au niveau national, de compagnies de régénération urbaine (Urban Regeneration Agencies) sur le modèle anglais que nous souhaitons aborder le thème de la tentative de «modernisation politique ».

\section{Le contexte}

A Glasgow, les responsables municipaux prennent conscience que les politiques urbaines à la dimension très fortement spatiale conduites à partir des années 1950 puis dans les années 1960 sont un échec urbanistique et politique majeur. Au début des années 1970, la ville entreprend de revoir sa stratégie d'aménagement sous l'influence du Scottish Office et d'une nouvelle génération de responsables. En 1972, à l'occasion de la deuxième révision du plan de développement de la ville ${ }^{3}$, l'utilisation d'indicateurs plus complets qu'auparavant aboutit au classement de la moitié de la ville dans les trois plus mauvais niveaux ${ }^{4}$. Cela prouve que le problème des quartiers défavorisés s'étend bien au-delà des vieilles zones de taudis traditionnellement étiquetées comme telles, c'est-à-dire les vieux quartiers du centre ville, pour concerner aussi un grand nombre des zones de logements sociaux construits et gérés par la municipalité après guerre. En d'autres termes, c'est toute la politique d'aménagement de la ville mise en place dans les années 1950 et 1960 par la municipalité travailliste qui montre ses limites et qui est pointée du doigt : c'est un échec social, environnemental et politique. Les grands ensembles en périphérie de la ville, quasiment uniquement des logements sociaux gérés par la municipalité travailliste qui avait pris le parti de pratiquer des loyers très modérés (avec quelques arrières pensées politiciennes), se sont considérablement dégradés ${ }^{5}$ et leurs populations ne s'y sont pas adaptées. Il faut dire que la construction des écoles, des magasins et des équipements publics n'est intervenue que bien après et qu'il s'agissait encore de lieux à très forte densité de population à cette époque. Ils poseront donc d'importants problèmes économiques, sociaux et environnementaux dans les années 1980.

C'est en fait toute la politique de déplacement de population et de création de villes nouvelles des années 1950 et 1960 qui est mise en cause. Cette politique avait été mise en place par le Clyde Valley Regional Plan de 1949. Toutefois, elle avait été largement édulcorée dans la pratique et n'était finalement à l'origine que de 6500 départs annuellement, contre une prévision de 10 000. Les départs spontanés de la ville s'élevaient quant à eux à 18500 par an. Globalement, les pertes de population ont largement dépassé les prévisions. Glasgow s'est littéralement vidée au cours des années 1960. A cette période, 25000 personnes quittent la ville chaque année, pour la plupart des ouvriers qualifiés et des professions libérales. De plus, ces personnes ne vont pas s'installer en périphérie mais elles quittent une région où il est devenu très difficile de (re)trouver

\footnotetext{
3 Glasgow, Second Review of the Development Plan. Areas in Need in Glasgow, R. D. Mansley, Director of Planning, 1972.

4 Les critères qui avaient été utilisés pour la rédaction du Plan de développement de 1960 sont étendus (Ils avaient conduit les autorités à identifier 4000 acres de logements surpeuplés et insalubres qui nécessitaient une rénovation complète - comprehensive redevelopment) Pour le Plan de développement de 1972, sont alors pris en compte pour la définition des zones défavorisées (multiple deprivation areas) les critères suivants: la composition des ménages, le chômage, les catégories sociales des résidents, le nombre de personnes retraitées, les conditions de logement, le taux d'occupation des logements et la taille des ménages qui y résident. L'analyse des données permet un classement des quartiers au niveau national selon six niveaux.

5 La municipalité n'avait pas les moyens de leur maintenance en raison des faibles loyers qu'elle percevait et de l'immensité de son parc de logements: Glasgow avait en son temps le plus grand parc de logements publics d'Europe.
} 
un emploi. De plus de un million d'habitants en 1951, la population de la ville tombe à 774000 en 1981.6

De même, l'activité industrielle qui s'y était implantée provenait d'ailleurs, alors qu’il était prévu que ces villes nouvelles soient le lieu de réimplantation de l'activité industrielle et de la population de Glasgow. Ainsi, entre 1958 et 1968, seuls 20.1\% des emplois qui quittent Glasgow se relocalisent dans une ville nouvelle. ${ }^{7}$ Finalement, le West Central Scotland Plan ${ }^{8}$ recommande la suspension des travaux concernant la ville nouvelle de Stonehouse le temps que le conseil régional de Strathclyde réfléchisse à la nécessité de poursuivre la politique de déplacements de population. Dans la pratique, cela mit un terme au rôle des villes nouvelles d'accueillir les populations déplacées de Glasgow.

Le contexte plus global dans lequel il faut replacer la ville au niveau économique pour comprendre la situation à la fin des années 1970 montre que le déclin industriel et économique de Glasgow s'est accéléré après la Seconde Guerre Mondiale qui, en restaurant le plein emploi sur les chantiers navals, avait réussi à dissimuler pour un temps encore les faiblesses structurelles de l'industrie lourde et des chantiers navals et à éluder les questions de stratégie industrielle. Après la guerre, elle ne résiste plus à la concurrence d'autres pays hors d'Europe. Glasgow subit alors une désindustrialisation vaste et très rapide. Les nouvelles industries ne parviennent pas à compenser les pertes d'emplois dans les usines de sidérurgie, de la construction mécanique et dans les chantiers navals qui ferment les uns après les autres. Elles préfèrent s'installer en périphérie, ou quitter la région, loin de l'emplacement de l'industrie lourde traditionnelle. Ce sont les hommes peu qualifiés qui exercent des métiers manuels qui sont les plus touchés. Entre 1961 et 1991, l'emploi dans l'industrie dans la conurbation passe de 387000 à $121000^{\circ}$. A titre d'exemple : en 1961, 38416 personnes sont employées dans les chantiers navals. Vingt ans après, elles ne sont plus que 12750 et il n'y a plus que 2 chantiers navals en activité dans la ville. Le nombre d'employés dans la métallurgie passe de 39195 à 16246 au cours de la même période. Dans l'industrie mécanique, la chute est tout aussi dramatique : il n'y a plus que 33086 employés dans ce secteur en 1981, contre 86467 en 1961'10. Parallèlement, l'emploi dans les services augmente peu, sauf pour les femmes. Il en résulte que les créations d'emplois dans le secteur des services sont loin d'être suffisantes pour absorber la perte d'emplois dans le secteur industriel. En valeur relative pourtant, on assiste à un renversement spectaculaire de la nature des emplois en vingt ans. La part des emplois dans les services passe de $42 \%$ en 1961 à $52 \%$ en 1971 et $63 \%$ en 1981. En vingt ans, Glasgow est devenue un centre de services ${ }^{11}$ et a tourné le dos à son passé industriel. Les pertes d'emplois considérables des années 1960 et 1970 se poursuivent pendant la décennie 1981 - 1991 qui voit la ville perdre encore 11\% de ses emplois (et $44 \%$ de ses emplois dans l'industrie). Globalement, Glasgow perd un quart de ses emplois dans la période entre 1952 et 1987 pour atteindre un seuil historique de 352200 emplois en 1996.

Le problème des quartiers défavorisés tel qu'il apparait au cours des années 1970 souligne la particularité de Glasgow : être confrontée à la fois à un centre ville et une périphérie où l'habitat est très dégradé et où résident des populations très fortement défavorisées et dont les perspectives d'amélioration de la situation économique sont pratiquement inexistantes. A partir de cette date, et de la prise de conscience de l'important délabrement de l'habitat de la ville (17 000 logements sont en attente de destruction en 1971), les autorités de la ville commencent à privilégier un travail de réhabilitation (regeneration) et d'amélioration de vieux quartiers centraux de

\footnotetext{
6 Elle est actuellement d'environ 585000. Les projections prévoient 597000 à l'horizon 2016.

7 Keating, M., 1988, p. 28. Les villes nouvelles sont East Kilbride, Livingstone, Glenrothes et Cumbernauld.

8 West Central Scotland Plan Steering Committee, West Central Scotland Plan - A Programme for Action, Glasgow, 1974.

${ }^{9}$ Gómez, M., 1998, p. 107-8.

${ }^{10}$ Keating, M., 1988, p. 168-9.

${ }^{11}$ Lever, W. et Moore, C., 1986, p. 2-3.
} 
la ville. La rénovation (redevelopment), c'est-à-dire la construction de nouveaux logements en lieu est place d'anciens, en rognant sur la ceinture verte ou en aménageant des sites industriels en friche, reste tout de même considérée comme la meilleure option. La construction de logements neufs au centre ville par le secteur privé commence toutefois à cette période à être présentée comme une solution d'avenir.

C'est à cette époque que la municipalité enclenche une réflexion qui va l'amener à tourner définitivement le dos à son passé industriel et à progressivement utiliser la culture sous toutes ses formes comme vecteur de sa régénération économique à partir du début des années 1980. Le semi-échec que constitue le programme de régénération de la partie est de la ville - la plus sévèrement touchée par la désindustrialisation et la dépopulation - GEAR (Glasgow Eastern Area Renewal), dont les ressources sont diminuées avec l'arrivée de Margaret Thatcher au pouvoir (comme les ressources généralement allouées aux organismes publics) est en ce sens significatif de la transition d'un modèle à un autre. GEAR débute en 1977, c'est un partenariat public-public entre diverses agences publiques dont notamment la Scotland Development Agency, Glasgow District Council, Strathclyde Regional Council et l'Office du Logement. L'objectif du programme GEAR était de coordonner l'action des diverses agences publiques dans le but de procéder au réaménagement du territoire sur lequel elles opéraient. Le programme a effectivement permis une amélioration sensible de l'habitat et de l'environnement. Plus de 50\% de l'argent investi dans l'East End de la ville fut en faveur du logement (70\% des logements appartenait à la ville). L'action de GEAR a été toutefois fortement critiquée car les conditions sociales et économiques se sont fortement dégradées à cette période et le programme n'a pas su (ou pu) dépasser le stade de l'amélioration de l'habitat et de l'environnement. Ce modèle de régénération urbaine, où l'intervention des agences publiques était coordonnée par une autre agence (en l'occurrence, ici, par une équipe issue de la Scottish Development Agency) avait peu de chance de se reproduire, étant donné le changement de contexte politique, la diminution de l'intervention étatique et, paradoxalement pour une administration centrale conservatrice prônant le moins d'Etat possible, le développement d'agences créées par le gouvernement central ${ }^{12}$.

\section{Les sociétés locales de développement}

La particularité de Glasgow est d'avoir été la première ville industrielle britannique à s'engager dans la voie de la régénération urbaine par la culture et à être désignée Capitale Européenne de la Culture en 1990. Les études ${ }^{13}$ soulignaient la nécessité pour la ville d'être plus attractive pour les entreprises et les personnes. Il fallait en priorité reconvertir les nombreuses friches industrielles, établir des lignes aériennes plus nombreuses en direction des grandes métropoles américaines et européennes et améliorer le cadre de vie pour encourager l'installation de sièges sociaux d'entreprises, le développement du tourisme d'affaire et de loisir, du commerce, des services et de l'industrie de haute technologie. Il fallait aussi rénover le centre ville pour le rendre attractif et vivant. Il était primordial de construire une image claire et positive de Glasgow. Pour atteindre ces objectifs, le développement d'une collaboration entre organismes publics et partenaires privés fut alors défini comme essentiel, et s'est traduit par l'émergence de sociétés locales de développement (local development companies) qui sont devenues depuis le $1^{\mathrm{er}}$ avril 2007 des agences de régénération locale (local regeneration agencies). Les sociétés locales de développement ont été des exemples de partenariats public-privé particulièrement en vue qui abordaient sous un angle global les divers aspects de la régénération de la ville dans les quartiers périphériques les plus en difficulté, à travers la mise en place de solutions intégrées dont l'objectif était d'intervenir à la fois sur l'ensemble des problématiques économiques, sociales, culturelles de ces quartiers et de leurs populations, tout en insistant très fortement sur l'importance de

\footnotetext{
${ }^{12}$ Lawless, P., 1989, p. 47-8.

${ }^{13}$ Le rapport McKinsey notamment, publié en 1985.
} 
l'investissement des communautés dans l'amélioration et la conservation de leur lieu de vie. Les élites de la ville, la presse ${ }^{14}$ et les grandes institutions internationales (OCDE et Union européenne) présentent ce processus de régénération comme une réussite exemplaire. Mais la recherche universitaire souligne que la ville n'a pas su résoudre ses problèmes sociaux, que seules les élites en ont bénéficié, que les emplois créés sont des emplois mal payés dans le secteur des services et que les problèmes économiques structurels de la ville n’ont pas été abordés ${ }^{15}$. Par ailleurs, les moyens considérables qui ont été consacrés à la régénération de la ville ont surtout été dévolus à la transformation du centre-ville et à l'amélioration de son image. En revanche, les zones périphériques n'ont pas joui de la même prodigalité et il est bien peu question des sociétés locales de développement dans l'évaluation de l'héritage à long terme des actions de régénération entreprises à Glasgow au cours des années 1980 puis 1990. Elles sont pourtant la mise en œuvre dans les zones périphériques d'un des principes fondateurs de la régénération urbaine à travers la culture: la collaboration entre acteurs publics et privés.

Fruits de la réflexion menée à partir des politiques urbaines conduites pendant les décennies précédents, huit sociétés locales de développement ont donc vu le jour entre 1986 et 2000: East End Partnership, Castlemilk Economic Development Agency, Drumchapel Opportunities, The Initiative, Greater Easterhouse Development Company, Govan Initiative, Glasgow North Limited et Greater Pollok Development Company. Elles correspondaient aux zones de la ville les plus défavorisées et les plus gravement touchées par le déclin économique qui avaient été reconnues « zones prioritaires de régénération » par la Glasgow Regeneration Alliance en 1993. Ces zones se situaient aussi bien à la périphérie de la ville (Castlemilk, Drumchapel, Easterhouse et Pollok) qu'autour du centre ville (Gorbals, Govan, East End et Glasgow North). Leurs objectifs était de

remédier à la pauvreté et à la polarisation sociale en procédant à une redistribution des ressources, des emplois, de la formation et des autres possibilités au profit des communautés désavantagées [...etet] de soutenir le développement économique au niveau local et de promouvoir la prospérité générale de la ville en valorisant les immeubles, le capital et les capacités entrepreneuriales inexploitées, de façon à créer des emplois, augmenter les revenus et améliorer les services. ${ }^{16}$

Les sociétés locales de développement couvraient un territoire comprenant $40 \%$ de la superficie de la ville, $60 \%$ de sa population totale (580 000 habitants en 2006) et $70 \%$ de la population en âge de travailler ${ }^{17}$.

Le rapport publié par l'Union européenne propose une analyse qui met en valeur un grand nombre d'aspects positifs. Le principal point développé est que les sociétés locales de développement ont permis une décentralisation des moyens et des politiques de lutte contre le déclin économique et la misère sociale. Leur installation de proximité leur assure souplesse, créativité et adéquation aux circonstances locales dont ne peuvent se prévaloir des agences qui opèrent sur des territoires plus vastes. Les sociétés locales de développement mettent toutes en avant l'implication de la communauté dans la vie locale, ce que leur implantation locale rend plus évidente et plus stimulante. L'Union européenne ${ }^{18}$ souligne que l'obligation de diversifier les sources de revenus à cause des baisses de subventions publiques a pu stimuler la créativité et l'efficacité en matière de recherche de financements publics ou privés. Mais cette exigence vis-àvis des sociétés locales de développement pour qu'elles assurent l'investissement dans de

\footnotetext{
${ }^{14}$ Voir l'article de Beatriz García, « Deconstructing the City of Culture: The Long-Term Legacies of Glasgow 1990 » in Urban Studies, 42, 5-6, 2005, pp. 841-868.

${ }^{15}$ García, B., 2005 et Mooney, G., 2004.

${ }^{16}$ Union Européenne, 2000, p.21.

${ }^{17}$ GCC, 2005, p. 5.

${ }^{18}$ Union européenne, 2000, p. 54.
} 
nouvelles activités, pour anticiper les opportunités commerciales, pour mettre au point des compétences de négociation tout en évitant les erreurs d'appréciation ne résiste pourtant pas à l'examen des budgets. Le financement est resté très majoritairement public. Les sociétés locales de développement étaient en concurrence pour l'obtention de subventions publiques. Les autorités locales et nationales se sont ainsi déchargées de leurs responsabilités vis-à-vis des populations en mettant en compétition des structures semi-publiques pour se partager des subventions publiques (limitant de fait la distribution de ressources) sous couvert de méthodes de gestion prétendument efficaces car empruntées au modèle de l'entreprise privée.

On peut ainsi défendre l'hypothèse que les sociétés locales de développement ne disposaient finalement ni de réel pouvoir, ni de ressources propres suffisantes, leur existence étant conditionnée par les ressources publiques. Une partie de leur financement dépendait de leur capacité à convaincre de la pertinence de leurs projets. Seul le budget de fonctionnement était prévisible, mais il restait en moyenne inférieur à 10\% du budget global et il fut soumis à des réductions au début des années $2000^{19}$.

Il apparait que les financements étaient soumis aux inflexions politiques, aux changements de gouvernants et aux priorités des bailleurs. De manière générale, les sociétés locales de développement sont restées relativement fragiles financièrement et vulnérables face aux dysfonctionnements de leurs principaux bailleurs ${ }^{20}$. La réalisation des projets locaux est restée dépendante dans une certaine mesure des priorités nationales, même si cela fut moins prégnant que dans un système complètement centralisé. Les différentes sociétés locales de développement se sont engagées, par la force des choses, dans une relation de concurrence ce qui a accru le risque de fragmentation et d'inégalité de la politique sociale et économique ${ }^{21}$. En conséquence, l'efficacité de ces dispositifs fut variable, en fonction notamment de l'investissement des populations, des dirigeants et de la situation de départ.

En termes d'aide aux chômeurs et aux entreprises et de développement de zones d'activités, les résultats publiés par les sociétés locales de développement dans leurs rapports d'activité annuels amènent à penser que leur action fut forcément positive. Mais les indicateurs économiques du recensement de 2001 montrent que les difficultés persistent. Les sociétés locales de développement ont subi les lois du marché mondial. Elles n’ont par exemple pas empêché la délocalisation des centres d'appel que la ville avait réussi à attirer au milieu des années 1990 dans des lieux plus compétitifs ${ }^{22}$ ni que $50 \%$ des emplois de la ville soient tenus par des non-résidents. Elles n'empêchent pas non plus le nombre d'emplois d'atteindre son plus bas niveau en 1996 avec 352200 emplois. ${ }^{23}$ Le nombre de résidents sans emploi est officiellement évalué à 21000 en 2008. Il y a de fortes chances qu'il s'agisse d'une sous-estimation après les effets de la crise et parce qu'un grand nombre de personnes sans emploi n'apparaissent pas dans les statistiques officielles pour tout un nombre de raisons. Il est des endroits où l'espérance de vie régresse : ainsi, à Shettleston dans l'East End, la circonscription la plus pauvre du Royaume-Uni, l'espérance de vie des hommes est-elle de 63 ans, soit dix ans de moins que la moyenne nationale et quatorze de moins que la moyenne britannique. Seize des zones les plus défavorisées d'Écosse se trouvent à Glasgow : 41\% des ménages de Glasgow vivent dans la pauvreté et, dans certains quartiers, c'est le cas de plus de $80 \%$ des enfants ${ }^{24}$. Ce constat alarmant est confirmé par une récente dépêche de l'AFP reprise sur le site de France 24 : le quartier de Calton, dans l'East End de la ville, détient le record de l'espérance de vie la plus basse d'Europe ${ }^{25}$.

\footnotetext{
${ }^{19}$ GCC, 2005, p. 22.

${ }^{20}$ Union Européenne, 2000, p. 64.

${ }^{21}$ Union Européenne, 2000, p. 21.

${ }^{22}$ Mooney, G., 2004, p. 335.

${ }^{23}$ Il est depuis remonté à 417900 en 2007.

${ }^{24}$ Mooney, G., 2004, p. 336.
} 
En dernière analyse, il est nécessaire de situer le travail des sociétés locales de développement dans le contexte global de la régénération de la ville. Kantor décrit Glasgow comme une ville avec une stratégie de régénération urbaine à deux vitesses ${ }^{26}$ : une avalanche de moyens est consacrée au centre-ville pour en faire la vitrine d'une capitale culturelle, au détriment des populations modestes pour lesquelles les sociétés locales de développement firent la course aux subventions publiques et aux investissements privés et organisèrent des programmes d'aide en faveur des laissés pour compte. C'est donc l'esprit dans lequel les sociétés locales de développement ont été mises en place qui est le plus sujet à caution. Il s'agissait d'agences aux moyens limités au regard des difficultés de tous ordres des quartiers où elles opéraient. C'est en fait une vitrine qui masque une réalité beaucoup moins séduisante. Sous couvert d'actions de proximité et de participation citoyenne, les autorités municipales se sont défaussées de leurs responsabilités. Leur rôle semble finalement avoir consisté à distiller de maigres ressources (au regard des difficultés colossales auxquelles sont toujours confrontés les anciens quartiers ouvriers et les développements urbains en périphérie) qu'elles obtenaient quasi-exclusivement d'institutions publiques.

Les sociétés locales de développement ont vraisemblablement amélioré le quotidien et donnent à penser que les quartiers défavorisés de la ville n'étaient pas complètement abandonnés. Mais une bonne partie de la population en difficulté n'a pas réussi le changement de cap d'une économie industrielle vers une économie de services et se trouve dans l'incapacité de se faire une place sur le marché de l'emploi. Rappelons que plus de la moitié des emplois de la ville sont tenus par des personnes qui n'y résident pas. Les sociétés locales de développement ont fourni une aide précieuse, avec leurs moyens, mais sans jamais inverser la tendance.

Les sociétés locales de développement n'intervenaient pas dans le domaine du logement social. La politique de construction et de rénovation de logements à Glasgow privilégie l'accession à la propriété, ce qui n'est pas à la portée des locataires des logements sociaux des quartiers périphériques. Les associations pour le logement jouent un rôle significatif dans le renouvellement et l'entretien des logements du parc public de la ville, de même que les coopératives qui visent à impliquer les résidents dans la gestion de leurs logements pour les inciter à respecter leur habitat. Significativement, la municipalité a opté pour le transfert de son parc de logements sociaux à la Glasgow Housing Association (GHA) en 2002, avec un effacement des dettes à la clé et des promesses d'améliorations importantes de la qualité de l'habitat. Cette action est fortement ressentie comme une privatisation ${ }^{27}$ et un désengagement de la ville face à ses responsabilités envers les populations modestes et en difficulté, même si elle reste représentée dans le directoire de GHA. La démunicipalisation des logements sociaux suscite d'ailleurs de sérieuses inquiétudes dans la population.

\section{Quelle modernisation de l'action publique dans la régénération urbaine à Glasgow et en Écosse?}

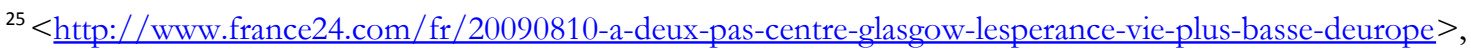
consulté le 25 septembre 2009.

${ }^{26}$ Kantor, P., 2000, p. 807-8.

${ }^{27}$ Mooney, G. et Poole, L., 2005, p. 34-37.
} 


\title{
La régénération à l'échelle locale : des Local Development Companies aux Local Regeneration Agencies
}

Les sociétés locales de développement ont laissé la place aux agences de régénération urbaine en avril 2007. Ces agences de régénération urbaine sont désormais au nombre de cinq et elles couvrent l'ensemble du territoire de la ville ${ }^{28}$. Elles ont pour mission de coordonner la déclinaison locale de la stratégie de régénération économique, sociale et physique de la ville, grâce à un travail en réseau avec les autres acteurs institutionnels tels que notamment Glasgow City Council, the Scottish Executive, Scottish Entreprise Glasgow et la NHS : "The five LRAs are independent companies, limited by guarantee with charitable status. Each provides a 'one-door' access route to an integrated range of economic development services within their own part of the city. $»^{29}$

La continuité en termes d'objectifs, de fonctionnement et de financement avec les sociétés locales de développement est évidente et, de façon très significative, le terme " régénération »a fait son apparition. Les intentions affichées par l'agence qui opère dans le sud ouest de la ville Glasgow South West Regeneration Agency - insistent par ailleurs clairement sur la prépondérance de la dimension économique du développement de son territoire :

\begin{abstract}
Improve the life of people living and working in the south west of Glasgow, strengthening communities, stimulate, develop, grow and sustain the area to make it an attractive place to live and work, getting people back to work, creating training opportunities, developing a strong and diverse business base, making communities safe and giving young people a positive route into education and employment.
\end{abstract}

Ces intentions s'inscrivent complètement dans la philosophie de renaissance urbaine définie par Richard Rodgers, qui a présidé l'Urban Task Force, dans le rapport remis par à Tony Blair en 1999 et devenu le véritable guide de la renaissance urbaine en Grande-Bretagne ${ }^{30}$.

\section{La régénération à l'échelle nationale: les Urban Regeneration Companies}

Dans le contexte plus large de l'Écosse, l'action publique menée par le gouvernement écossais dans le domaine de la régénération urbaine a elle aussi évolué. Des compagnies urbaines de régénération (Urban Regeneration Companies, URC) ont en effet vu le jour en 2004. Initialement au nombre de trois, elles sont progressivement passées à cinq puis six $^{31}$. Les URC ont pour objectif global d'être « an important opportunity to bring focus, integration, strong coordination

\footnotetext{
${ }^{28}$ Glasgow East Regeneration Agency, Glasgow South West Regeneration Agency, Glasgow West Regeneration Agency, Glasgow North Regeneration Agency et Glasgow South East Regeneration Agency. Le territoire sur lequel chacune d'elle opère recoupe celui des cinq Community Health and Care Partnerships (CHCP) créés à pareille époque. Chaque CHCP est lui-même subdivisé en deux Community Planning Partnerships (CPP). La ville est donc divisée en cinq grandes zones fonctionnelles dans lesquelles opèrent un Community Health and Care Partnership, deux Community Planning Partnerships et une agence de régénération urbaine. Ces zones fonctionnelles sont également valables dans le domaine de l'éducation.

$29<$ http://www.glasgow.gov.uk/en/AboutGlasgow/Factsheets/Glasgow/Urban+Regeneration.htm>, consulté le 25 septembre 2009.

${ }^{30}$ Urban Task Force, Towards an Urban Renaissance : Final Report of the Urban Task Force, London : E \& FN Spon, 1999. Pour une analyse de la renaissance urbaine en Angleterre telle qu'elle a été définie par Richard Rodgers et mise en œuvre par les différents gouvernements de Tony Blair depuis 1997, voir l'ouvrage collectif coordonné par D. Fée et S. Nail : Fée, D. et Nail, S., (sous la direction de), Vers une renaissance anglaise? Dix ans de politique travailliste de la ville, Paris, Presses Sorbonne Nouvelle, 2008.

${ }^{31}$ Les trois premières furent implantées en 2004 à Raploch (Stirling), Craigmillar (Édimbourg) et Clydebank, près de Glasgow. Les deux suivantes furent créées en 2006: il s'agit de Riverside Inverclyde (située le long de l'embouchure de la Clyde, de Port Glasgow à Greenock) et Irvine Bay (North Ayrshire). La dernière date de 2007 : il s'agit de Clyde Gateaway.
} 
and of course increased investment to areas in Scotland that have significant and complex regeneration needs. $~_{32}$ Plus précisément, le gouvernement écossais a défini les objectifs suivants pour les URC:

To provide a single vision and strategic focus for the regeneration of an area; To act as a catalyst for private sector investment by raising investor confidence; To guide investment by the public and private sectors towards an agreed set of objectives and outcomes; To speed up the pace of delivery; To maximise the use of public sector assets, including land; To provide a strategic approach to tackling infrastructure issues such as transport and land assembly. ${ }^{33}$

De même que l'influence du concept de renaissance urbaine défendu par Rodgers semble évidente dans l'évolution de la politique de régénération urbaine au niveau local à Glasgow, la mise en place des URC s'inscrit dans le contexte plus global de la politique de régénération urbaine mise en place par Tony Blair en Angleterre. Les URC ont en effet été proposées dans le rapport de l'Urban Task Force comme le moyen de mettre en œuvre la régénération des zones urbaines qui en avaient le plus besoin :

The pace of regeneration could be increased if responsibility for delivering area programmes was placed in the hands of 'arms length' organisations, owned by local partnerships. Urban Regeneration Companies (and Housing Regeneration Companies on similar lines) could not only oversee work to completion, but also raise private finance and undertake direct development where necessary ${ }^{34}$

C'est après avoir conduit une évaluation de l'action des URC en Angleterre que le gouvernement écossais a décidé de créer des agences identiques en Écosse. L'idée n'était alors pas de créer un dispositif innovant, mais un dispositif qui donne un second souffle à l'ensemble des agences ou dispositifs déjà existants, en apportant une coordination supplémentaire. On peut ainsi lire dans Review of Scotland's Cities :

URCs have therefore made a promising start in re-invigorating regeneration efforts in their designated areas in England and could offer potential for doing the same in Scotland were we to choose to adopt the approach. The URC approach offers little that is particularly new or unique in terms of a model. Indeed many of the success factors identified in the table can be demonstrated by a number of existing Scottish delivery vehicles. What the URC model does offer, however, is a renewed focus and dynamism for regeneration efforts, improved co-ordination of existing activities behind a comprehensive strategy and independence from local authorities and other public agencies. ${ }^{35}$

\footnotetext{
${ }^{32}$ Scottish Executive, 2007, p. 1

${ }^{33}$ Cité dans Scottish Executive, 2007, p. 4.

34 Urban Task Force, Towards an urban renaissance: report of the Urban Task Force - executive summary, p.12. http://www.eukn.org/binaries/united-kingdom/bulk/policy/2005/10/towards-an-urban-renaissance--finalreport-of-the-urban-task-force.pdf, consulté le 25 septembre 2009. Les principales recommandations formulées dans le rapport concernant la mise en place (delivering) de la politique de régénération sont: Create designated Urban Priority Areas, enabling local authorities and their partners in regeneration, including local people, to apply for special packages of powers and incentives to assist neighbourhood renewal; Strengthen the New Commitment to Regeneration between central and local government; Enable Urban Regeneration Companies and Housing Regeneration Companies to co-ordinate or deliver area regeneration projects (Urban Task Force, Towards an urban renaissance: report of the Urban Task Force - executive summary, p.12).

${ }^{35}$ Scottish Executive, 2002, p. 252.
} 
The Cities Review team therefore believes that there is potential in introducing key elements of the URC approach to Scotland, to provide a new impetus for regeneration efforts in areas of our cities where existing initiatives are failing to deliver. In particular, we believe there to be benefit in the role that the designation of URCs by central government plays in identifying national priorities and signalling to the private sector the intention of public agencies to focus efforts and investment to drive forward regeneration in particular areas. ${ }^{36}$

Les URC suivent toutes les mêmes objectifs, tels qu'ils ont été définis par le gouvernement écossais. Bien que toutes similaires dans leur façon de fonctionner, elles n'en ont pas moins leurs spécificités en fonction du contexte dans lequel elles opèrent. En suivant les recommandations formulées par Richard Rodgers dans le contexte anglais,

each company has worked to establish a regeneration strategy or framework, which had been followed by a spatial plan/ masterplan. The relevance of the former is to set a socio-economic context for the area, whereas the latter sets out a spatial development framework for its regeneration, that is based on that context and the needs/ opportunities articulated therein. ${ }^{37}$

Glasgow et toute la conurbation du centre ouest de l'Ecosse sont très largement concernées par l'établissement d'URC. L'URC Clyde Gateaway ${ }^{38}$ fut créée en décembre 2007, précédée par Clydebank en 2004 et Riverside Inverclyde en 2006. Si les deux dernières ne sont pas situées dans les limites administratives de Glasgow, elles font néanmoins partie de la conurbation glaswégienne.

L'East End de Glasgow contient parmi les zones les plus défavorisées d'Écosse et soufre d'un déclin économique, physique et social persistent. La population décline plus vite que le reste de la région, l'activité économique est très basse et le niveau d'emploi est également très bas. Cette zone de la ville contient 30\% des terrains en friche pollués de Glasgow et du Lanarkshire du Sud, situation aggravée par des contraintes importantes de drainage, d'infrastructure et de contamination. ${ }^{39}$

L'ensemble s'inscrit en partie dans la stratégie globale voulue par le gouvernement écossais qui a désigné la Clyde comme zone prioritaire de régénération urbaine sous l'appellation Clyde Corridor. Cette zone comprend le centre ville, et les deux parties respectivement en aval, le Clyde Waterfront ${ }^{0}$ (où se situe l'URC Clydebank) et, en amont, le Clyde Gateaway (où opère l'URC du même nom $)^{41}$.

\footnotetext{
${ }^{36}$ Scottish Executive, 2002, p. 253.

${ }^{37}$ Scottish Executive, 2007, p. vi.

${ }^{38}$ Les institutions partenaires sont Glasgow City Council, South Lanarkshire Council, Scottish Enterprise, Communities Scotland et the Scottish Executive. Le territoire couvert par l'URC Clyde Gateaway s'étend de Bridgeton Cross à Parkhead Cross et Farme Cross. Un des quartiers principaux est Dalmarnock, c'est-à-dire la partie de l'East End de Glasgow située au sud de London Road. Les objectifs de l'URC sont les suivants : to provide the long term certainty needed by private sector investors. Over the next 20 years, the targets include increasing the population of the area by 20,000, providing 10,000 new homes, generating 400,000 square metres of business space resulting in 20,000 jobs and remediating 350 bectares of vacant and contaminated land.

< http://www.glasgow.gov.uk/en/AboutGlasgow/Factsheets/Glasgow/Urban+Regeneration.htm $>$, consulté le 25 septembre 2009.

${ }^{39}$ Scottish Executive, 2007, p. 31.

40 Le Clyde Waterfront Plan est un vaste programme de régénération qui concentre 1,67 milliards de livres d'investissements et qui prévoit le développement de front de 32 projets majeurs sur une surface de 300 hectares dans les 15 années à venir. C'est en tout cas cette partie de la ville qui regroupe la majeure partie des infrastructures culturelles de tourisme et de loisirs. Il s'y construit aussi un nombre important de logements destinés au secteur privé.

${ }^{41}$ Voir Scottish Executive, Edinburgh, 2002.
} 
Il existe enfin un dernier niveau d'intervention : celui de la ville région. A la suite de la publication de Review of Scotland's Cities en 2002, les six principales villes écossaises ont dû produire un plan d'action à l'échelle de la ville région pour les dix années à venir (2003-2013), sans que cela soit pour autant une révolution puisque dans le cas de Glasgow, l'aire géographique couverte par la ville région reste identique à celle du plan régional précédent ${ }^{42}$. Contrairement aux dispositifs, actions et projets précédents qui visent tous à une régénération économique, physique, sociale la plus intégrée possible, en mettant l'accent sur la qualité de vie, c'est la dimension physique de la régénération qui prévaut.

\section{Les Jeux du Commonwealth de 2014: quelle continuité dans la stratégie d'aménagement urbain de Glasgow?}

Pour comprendre l'importance de l'URC Clyde Gateaway dans la stratégie globale de régénération de Glasgow, revenons sur le contexte de l'organisation des grandes manifestations culturelles ou sportives nationales et internationales à Glasgow. Cette démarche fait partie intégrante du projet de la ville entrepreneuriale, tournant que Glasgow a pris au début des années 1980 et génère en retour une compétition interurbaine de plus en plus féroce dont l'effet le plus patent est une sorte de fuite en avant vers la création de toujours plus d'événements culturels.

Glasgow s'est donc aussi engagée dans cette spirale événementielle, avec, comme nous l'avons évoqué précédemment, la particularité d'être une des premières villes industrielles à en saisir le potentiel. Les trois événements fondateurs de cette nouvelle politique ont été l'ouverture de la Burrell Collection en 1983, la réhabilitation de Merchant City, un quartier d'entrepôts du centreville transformé en logements et centre commercial et le lancement de la campagne promotionnelle "Glasgow's Miles Better» en 1982. Puis Glasgow a été l'hôte du Garden Festival en 1988, European City of Culture en 1990 et UK City of Architecture and Design en 1999 (pour ne citer que les événements les plus significatifs, au milieu d'une pléiade de festivals et autres manifestations annuels ou plus ponctuels organisés depuis cette période). Glasgow lance aussi périodiquement des campagnes promotionnelles plus ou moins agressives pour vendre son image et relayer les événements en cours ou à venir. Les plus significatives sont: "There's a lot Glasgowing on 》 (1990), "Glasgow's Alive » (1991), " Glasgow, The Friendly City 》 (1997), "Glasgow: Scotland with Style » (depuis 2004 et toujours d'actualité). Chacune de ces manifestations a été pour la ville l'occasion de se doter d'infrastructures à usage culturel et touristique (salles de spectacles, de conférences, hôtels) ou d'en rénover d'anciennes.

C'est dans ce contexte d'organisation d'événements festifs d'ampleur au service de la promotion et de la prospérité de la ville que Glasgow accueillera les Jeux du Commonwealth en 2014, malgré les réserves d'un certain nombre d'artistes ou d'universitaires qui critiquent ouvertement l'instrumentalisation des événements festifs au service de l'économie et la marchandisation de la ville. Ces Jeux du Commonwealth ont pour but de donner un souffle nouveau à l'économie de la ville et promouvoir une image la plus positive possible dans le monde entier, si l'ont veut bien croire Jack McConnell, le Premier Ministre écossais - travailliste - en poste lors de l'annonce du programme sportif des Jeux en janvier 2007:

Today's announcement is about more than confirming the sports that will be in the Games - it is about looking into the future and imagining what those Games will look like. In those eleven days, sport would transform a city, inspire a country and enthral millions of people. Glasgow 2014 will be an event like no other, one that the whole world will want to be part of. ${ }^{43}$

\footnotetext{
${ }^{42}$ Le nouveau plan s'intitule Metropolitan Glasgow. Le Glasgow and Clyde Valley Structure Plan qui le précédait, englobait les huit autorités locales suivantes: Glasgow, Inverclyde, Renfrewshire, East Renfrewshire, West Dunbartonshire, East Dunbartonshire, North Lanarkshire and South Lanarkshire.

${ }^{43}$ Cité sur <www.glasgow2014.com/Our-Bid/The-story-so-far/>, consulté le 25 septembre 2008.
} 
Cette emphase du discours politique trouve un écho dans l'introduction aux deux pages de la Glasgow Economic Review de juin 2006 consacrées aux Jeux du Commonwealth.

Bringing the 2014 Commonwealth Games to Glasgow would change our City. Hosting the world's second largest multi-sport event would make sure that the reinvention of Glasgow not only continues, but steps up a gear. It would bring jobs and investment. It would see regeneration and renewal in some of the communities that need it most. And it would showcase everything that our wonderful City has to offer to a global audience of billions. ${ }^{44}$

Glasgow avait commandé une étude pour analyser les éventuelles retombées économiques des Jeux du Commonwealth. L'étude prévoit la création de 1200 emplois en Écosse, dont 1000 à Glasgow, une augmentation de 4\% du nombre de visiteurs dans la ville pour un gain de 30 millions de livres dans les trois années qui suivent (GCC, 2006, 10-11). Enfin, l'étude souligne la contribution des Jeux du Commonwealth à l'amélioration de la qualité de la vie, de la motivation de la main d'œuvre et de l'image renvoyée aux investisseurs étrangers ainsi que dans le bouillonnement de la ville, tout en reconnaissant que tout cela reste difficilement quantifiable (!) (GCC, 2006, 11), le tout relayé par une intense communication des autorités qui ont acquis une expérience importante dans ce domaine:

All our efforts to improve lives can be crystallised in this one single event. Glasgow is a City on the move, full of people with a spring in their step. Bringing the Friendly Games to the Friendly City would make sure that Glasgow can indeed flourish. ${ }^{45}$

Le village des athlètes se situera à Dalmarnock, dans l'East Est de Glasgow, à environ 3 kilomètres du centre-ville. Le village sera construit de toutes pièces sur un site de 35 hectares avec un budget de 229 millions de livres sterling. La construction sera prise en charge par un partenariat public-privé ${ }^{46}$ et il sera situé à l'endroit où avait été érigé dans les années 1960 un ensemble comprenant 4 tours de 22 étages (toutes démolies entre 2002 et 2007) et des constructions plus basses (H-block maisonnettes). Le village pourra accueillir 8000 athlètes et officiels, avec des installations leur permettant de se préparer et s'entraîner sur place pour les compétitions. Il est prévu que ces logements soient transformés après les Jeux en logements pour 6000 à 6500 futurs résidents qui seront en grande majorité (1200 logements sur les 1500 construits) destinés au marché immobilier privé (locatif ou propriétaires occupants), le reste revenant aux associations gestionnaires de logements sociaux. On peut affirmer sans grand risque d'erreur qu'il y a peu de chance que la population locale, dont $54 \%$ vivent actuellement dans des logements sociaux, pourra s'acheter un logement sur le site des Jeux du Commonwealth, d'autant plus que l'objectif est d'attirer 20000 habitants dans cette zone de la ville dans les 25 prochaines années. Il s'agit actuellement du plus important projet de construction de logements de la ville qui vient compléter le programme de régénération économique et de désenclavement de l'est de la ville porté par l'URC Clyde Gateaway. La construction d'un tronçon d'autoroute (M74) qui traverse le sud de la ville pour relier l'autoroute qui dessert l'ouest de la ville et le centre ville à l'autoroute qui dessert l'est de la ville et d'un axe de circulation majeur (East End Regeneration Route) en sont les deux autres projets phares.

\section{Conclusion}

\footnotetext{
44 Glasgow City Council, 2006, p. 10.

45 Glasgow City Council, 2006, p. 11.

${ }^{46}$ Glasgow 2014, 2007, p. 131-2.
} 
La régénération des anciennes zones industrielles reste un problème majeur en Écosse, à la hauteur des dégâts causés par la désindustrialisation sur le tissu humain et économique et l'environnement. À travers l'exemple de Glasgow et, plus largement, de tout le centre ouest de l'Écosse, on s'aperçoit que les modalités de l'action publique dans ce domaine évoluent constamment. La régénération se veut la plus complète et la plus intégrée possible, au risque d'y perdre toute lisibilité. La modernisation de l'action publique prend en effet la forme d'un entrelacs d'acteurs publics et privés, de dispositifs, d'actions et de plans qui sont constamment évalués, mis à jour, qui font l'objet d'une intense communication, et de très nombreuses publications institutionnelles. La multiplication des structures, agences et compagnies pose nécessairement la question de leur pilotage, local ou national, ainsi que la définition de leurs missions et de leurs zones de compétences et d'intervention. Enfin, cette modernisation n'a rien de très innovant : la filiation avec ce qui se passe "south of the border » est évidente, quand elle n'est pas clairement revendiquée.

Glasgow a indéniablement opéré une vraie métamorphose grâce notamment à l'organisation d'événements festifs d'ampleur nationale et internationale. L'image qu'elle donne à voir aux visiteurs a radicalement changé et elle a définitivement tourné le dos à son passé industriel. Tout au plus en a-t-elle conservé la meilleure partie de son héritage architectural avec la réhabilitation des immeubles victoriens ou la conversion de certains entrepôts désaffectés du centre-ville en logements, centres commerciaux ou salles de spectacles. L'imposante Finnieston Crane, déplacée aux portes du centre-ville rappelle aussi son passé d'excellence dans le domaine des chantiers navals. Le People's Palace, situé dans Glasgow Green, le parc où bien souvent les ouvriers se rassemblaient pour manifester lors de la grande période radicale socialiste propose quant à lui une exposition permanente sur l'histoire sociale (et donc ouvrière) de la ville. Mais tout ceci ressemble aux vestiges d'un lointain passé. La tradition ouvrière radicale de Red Clydeside (1910-1930) semble bien lointaine maintenant, même si pour Mitchell (2005) Glasgow reste probablement la seule ville britannique où subsistent encore une véritable culture et conscience ouvrières.

Glasgow est ainsi (re)devenue une capitale économique et culturelle de dimension internationale. Le centre-ville a été nettoyé, aseptisé et formaté pour les besoins de l'économie globale. Les quartiers réhabilités du centre-ville et des rives de la Clyde sont touchés par la gentrification, malgré la volonté de mixité sociale affichée par les autorités locales. Les services constituent désormais la majeure partie de son activité économique mais n'offrent pas toujours des emplois stables, qualifiés et bien rémunérés. L'activité industrielle est devenue presque anecdotique. La bonne santé du tourisme de loisir ou d'affaire est la clé de la réussite économique de nombreux secteurs d'activité de la ville. Mais c'est un secteur extrêmement concurrentiel. Glasgow connaît une croissance supérieure à toutes les villes importantes du Royaume-Uni et fait partie du peloton de tête des villes européennes de taille identique. Combien de temps cela peut-il durer? Glasgow n'est pas à l'abri d'un phénomène de saturation et que l'offre finisse par dépasser la demande. Récemment, Glasgow n’a pas été épargnée par la crise.

Les politiques de régénération urbaine mises en œuvre à Glasgow et dans sa conurbation depuis une trentaine d'années oeuvrent à la réalisation d'un pari presque impossible : développer prospérité et développement économique tout en essayant de combattre les problèmes sociaux hérités de la désindustrialisation. Dans un contexte de concurrence globale entre les villes, quand le centre ville et la Clyde sont l'objet de toutes les attentions et des principaux investissements, il apparait souvent que cela se manifeste par des phénomènes de polarisation sociale qui ne font que s'accroître.

\section{Bibliographie:}


Bidou-Zachariasen, C., (sous la direction de), Retours en ville. Des processus de "gentrification" urbaine aux politiques de "revitalisation” des centres, Paris, Descartes \& Cie, 2003.

Cunningham-Sabot, E., «Polycentrisme et gouvernance dans la Central Belt, Écosse » in Espace géographique, 36, 4, 2007, p. 304-319.

Donnison, D. et Middleton, A., (sous la direction de), Regenerating the Inner City - Glasgow's Experience, London, Routledge and Kegan Paul, 1987.

Fée, D. et Nail, S., (sous la direction de), Vers une renaissance anglaise? Dix ans de politique travailliste de la ville, Paris, Presses Sorbonne Nouvelle, 2008.

Friend of Zanetti, "Constructing Neoliberal Glasgow: The Privatisation of Space » in Variant, 25, 2006, p. 19-22.

García, B., «Urban Regeneration, Arts Programming and Major Events: Glasgow 1990, Sydney 2000 and Barcelona 2004" in International Journal of Cultural Policy, 10, 1, 2004, p. 103-116.

García, B., «Cultural Policy and Urban Regeneration in Western European Cities: Lessons from Experience, Prospects for the Future » in Local Economy, 19, 4, 2004, p. 312-326.

García, B., «Deconstructing the City of Culture: The Long-Term Legacies of Glasgow 1990 » in Urban Studies, 42, 5-6, 2005, p. 841-868.

Glasgow City Council, Glasgow Local Development Company Network - Annual Review 2004-2005, Glasgow, Glasgow City Council, 2005.

Glasgow City Council, Glasgow Economic Review - June 2006, Glasgow, Glasgow City Council, 2006.

Glasgow City Council, Glasgow Economic Review - Nov 2007 - Special Edition - State of the City Economy, Glasgow, Glasgow City Council, 2007.

Glasgow 2014, People, Place, Passion. Glasgow 2014 Commonwealth Games Candidate City File, Glasgow, Glasgow 2014, 2007.

Gómez, M., "Reflective Images: the Case of Urban Regeneration in Glasgow and Bilbao ", International Journal of Urban and Regional Research, 22, 1, 1998, p. 106-121.

Gray, N., «The Clyde Gateaway: A New Urban Frontier» in Variant, 33, 2008, p. 8-12.

Gray, N., «Glasgow's Merchant City: An Artist Led Property Strategy » in Variant, 34, 2009, p. 14-19.

Jeannier, F., «La régénération des quartiers industriels sinistrés: le rôle des sociétés locales de développement à Glasgow » in Géocarrefour, 81, 2, 2006, p. 127-133.

Kantor, P., "Can Regionalism Save Poor Cities? Politics, Institutions and Interests in Glasgow» in Urban Affairs Review, 35, 6, 2000, p. 794-820.

Keating, M., The City that Refused to Die. Glasgow: The Politics of Urban Regeneration, Aberdeen, Aberdeen University Press, 1988.

Lawless, P., Britain's Inner Cities, London, Paul Chapman, 1989.

Lever, W. et Moore, C., The City in Transition: Policies and Agencies for the Economic Regeneration of Clydeside, Oxford, Clarendon Press, 1986.

Lever, F. W., "Deinsdustrialisation and the Reality of the Post-industrial City » in Urban Studies, 28, 6, 1991, p. 983-999.

McCrone, G., «Urban Renewal: The Scottish Experience » in Urban Studies, 28, 6, 1991, p. 919938. 
Mitchell, I. R., This City Now, Glasgow and its Working-Class Past, Edinburgh, Lutha Press Ltd, 2005.

Mooney, G., «Cultural Policy as Urban Transformation? Critical Reflections on Glasgow, European City of Culture $1990 »$ in Local Economy, 19, 4, 2004, p. 327-340.

Mooney, G. et Poole, L., « Marginalised Voices: Resisting the Privatisation of Council Housing in Glasgow » in Local Economy, 20, 1, 2005, p. 27-39.

OCDE, Urban Renaissance - Glasgow : Lessons for Innovation and Implementation, Paris, OCDE, 2002.

Palmer-Rae Associates, European Cities Capitals of Culture, Study Prepared for the European Union Part 1, Brussels, Palmer-Rae Associates, 2004.

Scottish Executive, Review of Scotland's Cities, Edinburgh, Scottish Executive, 2002.

Scottish Executive, Building Better Cities: Delivering Growth and Opportunities, Edinburgh, Scottish Executive, 2003.

Scottish Executive, Best Practice in Establishing Urban Regeneration Companies in Scotland, Edinburgh, Scottish Executive, 2007.

Urban Task Force, Towards an urban renaissance: report of the Urban Task. Force - executive summary, $<$ http://www.eukn.org/binaries/united-kingdom/bulk/policy/2005/10/towards-an-urbanrenaissance--final-report-of-the-urban-task-force.pdf $>$, accès le 25 septembre 2009.

Union Européenne, Inclusive Cities: Building Local Capacity for Development, Luxembourg, Office for Official Publications of the European Communities, 2000. 